\section{Research news in clinical context}

\author{
Francesca Ceccherini-Silberstein 다, ${ }^{1}$ Miguel Fernández-Huerta, ${ }^{2}$ \\ Anna Maria Geretti ${ }^{3}$
}

\section{USING CYTOKINE EXPRESSION TO DISTINGUISH BETWEEN ACTIVE AND TREATED SYPHILIS: PROMISING BUT NOT YET READY FOR PRIME TIME}

Distinguishing between previously treated and active syphilis can be challenging in the subset of treated patients with serofast status, defined as persistent non-treponemal seropositivity $(<4$ fold decline in rapid plasma reagin titre $\geq 6$ months after treatment). The study investigated whether serum cytokine expression levels, measured with a 62-cytokine multiplex bead-based ELISA, can help guide clinical management. Using samples from patients with active, treated and serofast syphilis, the authors developed a two-cytokine (brain-derived neurotrophic factor and tumour necrosis factor $\beta$ ) decision tree that showed good accuracy (82\%) and sensitivity $(100 \%)$ but moderate specificity $(45 \%)$. While further studies will be needed to confirm and refine the diagnostic algorithm, there also remain important technical, operational and financial barriers to implementing such cytokine assays in routine care.

Kojima N, Siebert JC, Maecker H, et al. The application of cytokine expression assays to differentiate active from previously treated syphilis. J Infect Dis. 2020 [published online ahead of print, 2020 Mar 19].

\section{GLOBAL AND REGIONAL PREVALENCE OF HERPES SIMPLEX VIRUS TYPE 2 INFECTION: UPDATED ESTIMATES FOR PEOPLE AGED 15-49 YEARS}

Estimates of genital herpes simplex virus (HSV) infections across regions inform advocacy and resource planning and guide the development of improved control measures, including vaccines. In 2016, HSV-2 affected $13 \%$ of the global population aged 15-49 years (high-risk groups excluded), totalling 491 million

\footnotetext{
'Department of Experimental Medicine, University of Rome Tor Vergata, Rome, Italy

${ }^{2}$ Department of Microbiology, Bellvitge University Hospital, L’Hospitalet de Llobregat, Spain

${ }^{3}$ Institute of Infection, University of Liverpool, Liverpool, UK
}

Correspondence to Professor Francesca CeccheriniSilberstein, University of Rome Tor Vergata, 00133 Roma, Italy; ceccherini@med.uniroma2.it people. Of note, by excluding people aged $>49$ years, the analysis knowingly underestimated the true burden of HSV-2 infection. ${ }^{1}$ Prevalence showed a slight increase relative to 2012 and was highest in Africa and Americas and among women. Given the association between HSV-2 and subsequent HIV infection, ${ }^{2}$ it is concerning that HSV-2 was estimated to affect $\sim 50 \%$ of women aged 25-34 years in the African region. The analysis also estimated the prevalence of genital HSV-1 (3\%), but uncertainty intervals were wide.

James C, Harfouche M, Welton NJ, et al. Herpes simplex virus: global infection prevalence and incidence estimates, 2016. Bull World Health Organ. 2020; 98: 315-329.

\section{OBSERVED PREGNANCY AND NEONATAL OUTCOMES IN WOMEN WITH HIV EXPOSED TO RECOMMENDED ANTIRETROVIRAL REGIMENS}

This large Italian observational cohort study analysed data from 794 pregnant women who were exposed within 32 weeks of gestation to recommended antiretroviral regimens in the period 2008-2018. Treatment comprised threedrug combinations of an nucleoside reverse transcriptase inhibitor (NRTI) backbone plus a ritonavir-boosted protease inhibitor (78\%, predominantly atazanavir), an non-NRTI (NNRTI) (15\%, predominantly nevirapine) or an integrase strand transfer inhibitor (INSTI; 6\%, predominantly raltegravir). No major differences were found for a wide range of pregnancy and neonatal outcomes, including major congenital defects. The rate of HIV transmission ranged up to $2.4 \%$ in this study. This comprehensive evaluation will be useful for clinicians caring for women with HIV. More outcome data are needed for regimens comprising second-generation INSTIs.

Floridia M, Dalzero S, Giacomet V, et al. Pregnancy and neonatal outcomes in women with HIV-1 exposed to integrase inhibitors, protease inhibitors and nonnucleoside reverse transcriptase inhibitors: an observational study. Infection 2020;48:249-258.

\section{HIV STATUS AND SEXUAL PRACTICE INDEPENDENTLY CORRELATE WITH GUT DYSBIOSIS AND UNIQUE MICROBIOTA SIGNATURES}

Gut dysbiosis may contribute to persistent inflammation in people with HIV (PWH) who receive antiretroviral therapy (ART). The study compared the gut microbiota of ART-treated PWH and HIV-negative controls matched for age, gender, country of birth, body mass index and sexual practice. Regardless of sex and sexual practice, the gut microbiota differed significantly in PWH vrsus controls, with expansion of proinflammatory gut bacteria and depletion of homeostasispromoting microbiota members. The extent of dysbiosis correlated with serum inflammatory markers, nadir and pre-ART CD4 cell counts, and prevalence of noninfectious comorbidities. Further studies are warranted to elucidate causality and investigate microbiota-mediated strategies to alleviate HIV-associated inflammation. Independent of HIV status, and in both men and women, receptive anal intercourse was associated with a unique microbiota signature.

Vujkovic-Cvijin I, Sortino O, Verheij E, et al. HIV-associated gut dysbiosis is independent of sexual practice and correlates with non-communicable diseases. Nat Commun. 2020;11:2448.

\section{REDUCING THE COST OF MOLECULAR STI SCREENING IN RESOURCE-LIMITED SETTINGS: AN OPTIMISED SAMPLE- POOLING ALGORITHM}

Infections with Chlamydia trachomatis (CT) and Neisseria gonorrboeae (NG) are frequently asymptomatic and, if untreated, may lead to severe reproductive complications in women. Molecular testing is highly sensitive but costly, especially for resource-limited settings. This modelling study explored a sample pooling strategy for CT and NG testing among women in Zambia. Based on cross-sectional data, participants were stratified into high, intermediate and low prevalence groups, and the respective specimens were mathematically modelled to be tested individually, in pools of 3 , or pools of 4 , using the GeneXpert instrument. Overall, the pooling strategy was found to maintain acceptable sensitivity (ranging from 80\% to $100 \%$ ), while significantly lowering cost per sample. Investigation in additional cohorts will validate whether the approach may increase access to STI screening where resourced are constrained.

Connolly S, Kilembe W, Inambao M, et al. A population-specific optimized 
GeneXpert pooling algorithm for Chlamydia trachomatis and Neisseria gonorrhoeae to reduce cost of molecular STI screening in resource-limited settings. $J$ Clin Microbiol. 2020 [published online ahead of print, 2020 Jun 10].

\section{GIRL-ONLY HPV VACCINATION CAN ELIMINATE CERVICAL CANCER IN MOST LOW AND LOWER MIDDLE INCOME COUNTRIES BY THE END OF THE CENTURY, BUT MUST BE SUPPLEMENTED BY SCREENING IN HIGH INCIDENCE COUNTRIES}

Progress towards the global elimination of cervical cancer must include effective interventions in lower-middle income countries (LMICs). The study modelled the effect over the next century of girlsonly human papilloma virus (HPV) vaccination with or without once-lifetime or twice-lifetime cervical screening in 78 LMICs, assuming 90\% vaccine coverage, $100 \%$ lifetime protection and screening uptake increasing from 45\% (2023) to $90 \%$ (2045 onwards). Vaccination alone would substantially reduce cancer incidence (61 million cases averted) and achieve elimination $(<5$ cases per 100000 women-years) in $60 \%$ of LMICs. However, high-incidence countries, predominantly in Africa, might not reach elimination by vaccination alone. Adding twice-lifetime screening would achieve elimination of cervical cancer in 100\% of LMICs. Results have informed the targets of $90 \% \mathrm{HPV}$ vaccination coverage, $70 \%$ screening coverage and $90 \%$ of cervical lesions treated by 2030 recently announced by the WHO.

Brisson M, Kim JJ, Canfell K, et al. Impact of HPV vaccination and cervical screening on cervical cancer elimination: a comparative modelling analysis in 78 lowincome and lower-middle-income countries. Lancet 2020;395:575-590.

Handling editor Anna Maria Geretti

Twitter Anna Maria Geretti @Prof Anna Maria Geretti@GerettiAnna

Contributors All authors contributed equally to the manuscript.
Competing interests None declared.

Provenance and peer review Commissioned; internally peer reviewed.

(c) Author(s) (or their employer(s)) 2020. No commercial re-use. See rights and permissions. Published by BMJ.

A) Check for updates

To cite Ceccherini-Silberstein F, Fernández-Huerta M, Geretti AM. Sex Transm Infect 2020;96:471-472.

Sex Transm Infect 2020;96:471-472.

doi:10.1136/sextrans-2019-054275

ORCID iD

Francesca Ceccherini-Silberstein http://orcid.org/00000002-6024-5101

\section{REFERENCES}

1 GBD 2017 Disease and Injury Incidence and Prevalence Collaborators. Global, regional, and national incidence, prevalence, and years lived with disability for 354 diseases and injuries for 195 countries and territories, 1990-2017: a systematic analysis for the global burden of disease study 2017 Lancet 2018:392:1789-858.

2 Looker KJ, Elmes JAR, Gottlieb SL, et al. Effect of HSV-2 infection on subsequent HIV acquisition: an updated systematic review and meta-analysis. Lancet Infect Dis 2017:17:1303-16. 